\title{
Novel three-IncRNA signature predicts survival in patients with pancreatic cancer
}

\author{
BIN WU $^{1 *}$, KAIXUAN WANG $^{2 *}$, JANGUO FEI $^{1}$, YI BAO $^{3}$, XIAOGUANG WANG $^{1}$, \\ ZHENGWEI SONG $^{1}$, FEI CHEN ${ }^{1}$, JUN GAO $^{2}$ and ZHENGXIANG ZHONG ${ }^{1}$ \\ ${ }^{1}$ Department of Hepatobiliary Surgery, The Second Hospital of Jiaxing, Jiaxing, Zhejiang 314000; \\ ${ }^{2}$ Department of Gastroenterology, Changhai Hospital, Second Military Medical University, Shanghai 200000; \\ ${ }^{3}$ Department of Central Laboratory, The Second Hospital of Jiaxing, Jiaxing, Zhejiang 314000, P.R. China
}

Received December 2, 2017; Accepted September 17, 2018

DOI: $10.3892 / o r .2018 .6761$

\begin{abstract}
A growing body of evidence confirms that long non-coding RNAs (lncRNAs) have an important role in biological processes by regulating gene expression at multiple levels. Dysregulated IncRNAs may be potential prognostic biomarkers or targets for the development of cancer treatments. However, the prognostic role of an lncRNA signature in pancreatic cancer has not been investigated. Pancreatic cancer lncRNA expression profiles from The Cancer Genome Atlas (TCGA) were analyzed in the current study. The prognostic value of differentially expressed lncRNAs (DElncRNAs) was evaluated via the Kaplan-Meier method. A risk score model was established based on the potential prognostic lncRNAs. The biological functions of IncRNAs were predicted by functional enrichment analysis. Then, an lncRNA-mRNA co-expression network was established and predicted the function of the lncRNAs. Seven DElncRNAs that were significantly associated with the prognosis of pancreatic cancer were identified. Patients were classified into high-risk and low-risk groups using a risk score based on a three-lncRNA signature. There was a significant difference in overall survival (OS) between the groups (median OS 1.33 vs. 3.65 years; log-rank test, $\mathrm{P}=0.0000$ ). Cox regression analysis and ROC curves demonstrated that the three-lncRNA signature may be an effective independent prognostic biomarker in patients with
\end{abstract}

Correspondence to: Professor Zhengxiang Zhong, Department of Hepatobiliary Surgery, The Second Hospital of Jiaxing, 1,518 North Huancheng Road, Nanhu, Jiaxing, Zhejiang 314000, P.R. China E-mail: 13505739209@139.com

Dr Jun Gao, Department of Gastroenterology, Changhai Hospital, Second Military Medical University, 168 Changhai Road, Yangpu, Shanghai 200000, P.R. China

E-mail: 13816012151@163.com

*Contributed equally

Key words: pancreatic cancer, long non-coding RNA, survival, signature pancreatic. The functional enrichment analysis showed that lncRNA AL137789.1, one component of the three-lncRNA signature, may be associated with tumor immune responses. In the present study, a novel three-lncRNA signature that was established that may be useful in predicting survival among patients with pancreatic cancer. These lncRNAs may be involved in tumor immunity and thus affect the prognosis of patients.

\section{Introduction}

Pancreatic cancer (PC) is one of the most malignant tumor types, with a 5-year overall survival rate of $<6 \%$ (1). Globally, pancreatic cancer is the fourth most common cause of cancer-associated mortality. By 2030, pancreatic cancer may surpass breast cancer as the second most deadly tumor in the US (2). Patients with pancreatic cancer with no obvious clinical symptoms are frequently not diagnosed until advanced tumor stages. In recent years, with the development of molecular diagnoses and targeted therapies, there has been a huge improvement in diagnosis and treatment. However, pancreatic cancer, as a highly heterogeneous disease, still lacks effective diagnostic and prognostic biomarkers (3). To improve the rate of early diagnosis and extend the survival time of patients with pancreatic cancer, it is essential to identify effective tumor biomarkers for early diagnosis and prognosis risk stratification. This will not only help clinicians choose effective and individualized treatment programs, it will also be conducive to future investigations into the molecular mechanisms of the occurrence and development of pancreatic cancer.

Due to the development of genomic and transcriptomic sequencing techniques, an increasing number of non-coding RNAs (ncRNAs), which differ from protein-coding RNAs, have been identified. ncRNAs are divided into two categories according to size: Short ncRNAs and long ncRNAs (lncRNAs). IncRNAs are defined as $>200$ nucleotides in length (4). The Human Genome Project has indicated that protein-coding genes account for $<2 \%$ of the total sequence of the genome (5). Our understanding of lncRNAs is also very limited because the number of ncRNAs, including lncRNAs, is huge. It has been reported that the majority lncRNAs do not have gene regulatory functions; however, 
there is growing evidence to support the idea that lncRNAs may regulate gene expression at multiple levels, including genomic imprinting and transcriptional and post-transcriptional regulation (6). Chen et al (7) demonstrated that lncRNA small nucleolar RNA host gene 20 promotes the proliferation and migration of lung cancer cells by silencing p21 expression. Additionally the lncRNA DPP10 antisense RNA 1 inhibits the metastasis of colorectal cancer by upregulating TIMP metallopeptidase inhibitor 3, which suggests that this lncRNA could be used as a new therapeutic target for colorectal cancer (8). In recent years, a novel mechanistic type of lncRNA, known as a competing endogenous RNA (ceRNA), has been reported (6). IncRNAs of this class harbor microRNA (miR) response elements and can compete with miRs to communicate with mRNAs and to regulate the expression of genes (9). The study demonstrated that the IncRNA colon cancer associated transcript 2 regulates miR-145 expression by inhibiting its maturation in colon cancer cells (10). Another study reported that long intergenic non-protein coding RNA 941 acted as a ceRNA for miR-34a, leading to Snaill upregulation and epithelial-mesenchymal transition activation in hepatocarcinogenesis (11).

Regarding pancreatic cancer, it has been reported that several lncRNAs, including HOX transcript antisense RNA (12), HOXA distal transcript antisense RNA (13), metastasis associated lung adenocarcinoma transcript $1(14,15)$ and long intergenic non-protein coding RNA, regulator of reprogramming (16), are differentially expressed between pancreatic cancer tissues and normal tissues. These findings indicate that lncRNAs may be potential diagnostic or prognostic biomarkers for pancreatic cancer. However, a single lncRNA biomarker is often limited in its diagnostic and prognostic predictive value. Recent studies have reported that several lncRNA signatures may improve prognosis predictions for certain malignant tumors, including lymphoma (17), colorectal (18), esophageal (19) and lung cancer (20). However, the prognostic role of IncRNAs in pancreatic cancer has not been investigated. To determine an effective signature of lncRNAs for predicting pancreatic cancer survival, IncRNA expression profiles from a large number of patients with pancreatic cancer were obtained and analyzed by repurposing the publicly available Cancer Genome Atlas (TCGA) database in this study.

\section{Materials and methods}

Data processing. The lncRNA expression data and corresponding clinical data of pancreatic cancer were obtained from the TCGA database (portal.gdc.cancer.gov/) using the search terms 'Project $=$ TCGA-PAAD'. The data included lncRNA expression profiles of 178 cancer tissue samples and 4 normal tissue samples from 177 patients with pancreatic cancer. Analysis of differentially expressed lncRNAs (DElncRNAs) between pancreatic tumor tissues and normal tissues was performed using the edger package (bioconductor. org/packages/release/bioc/html/edgeR.html) in R (software version 3.1.4; r-project.org) (21). IncRNAs satisfying the conditions $\log _{2} \mid$ fold change $(\mathrm{FC}) \mid>1.0$ and $\mathrm{P}<0.05$ were considered to be differentially expressed. Unsupervised hierarchical cluster analysis using the gplots package (cran.r-project.org/web/packages/gplots/index.html) in
$\mathrm{R}$ (software version 3.1.4; r-project.org) revealed that normal tissues could be distinguished from pancreatic cancer tissues based on the DElncRNA patterns. Unsupervised hierarchical cluster analysis evaluated the degree of variation of DElncRNA patterns from pancreatic cancer tissues and normal tissues.

Association of DElncRNAs with patient prognosis. Patients with no prognostic information were excluded and then combined the DElncRNA expression profiles with the corresponding survival prognostic information. The prognostic value of each DElncRNA was evaluated using a Kaplan-Meier curve and the log-rank method. Patients were divided into the high expression and low expression groups for each of the lncRNAs according to the median value of the expression. The DElncRNAs that were significantly associated with overall survival (OS) were identified as potential prognostic lncRNA biomarkers.

Cox regression analysis and the establishment of a prognostic risk score model. The potential prognostic lncRNAs obtained by Kaplan-Meier survival analysis were included in a Cox regression analysis. In the Cox regression analysis, it was attempted to identify the best signature associated with patient survival by using the least number of lncRNAs. Then, a prognostic risk model consisting of multiple pancreatic cancer lncRNAs was established, and a risk score was computed as

$$
\text { Risk score }=\sum_{i=1}^{N}(\mathrm{Ei} * \mathrm{Ci})
$$

follows:

Risk score $=\left(\mathrm{N}\right.$, the number of prognostic lncRNAs; $\mathrm{E}_{\mathrm{i}}$, the expression value of $\operatorname{lncRNA} A_{i} ; C_{i}$, the estimated repression coefficient of $\operatorname{lncRNA}_{i}$ in the multivariable Cox regression analysis). The risk score for each patient was calculated, and all patients were divided into two groups (a high-risk group and a low-risk group) according to the median risk score. The difference in prognosis between the high-risk group and the low-risk group was evaluated by the Kaplan-Meier method. Then, to evaluate the prognostic risk score model for the 3- and 5-year survival rates of patients with pancreatic cancer, the receiver operating characteristic (ROC) curve was analyzed. The area under the ROC curve (AUC) was calculated from the ROC curves.

Establishment of an lncRNA-mRNA co-expression network and lncRNA functional predictions by bioinformatics analysis. An lncRNA-mRNA co-expression network using the WCGNA package in $\mathrm{R}$. The co-expression relationships between the DElncRNAs and DEprotein-coding genes were calculated using Pearson correlation coefficients (the cut-off of Pearson correlation coefficient was $>0.6$ ). Gene Ontology (GO) and Kyoto Encyclopedia of Genes and Genomes (KEGG) enrichment analyses of the co-expressed protein-coding genes with DElncRNAs were performed to predict the biological function of DElncRNAs using the DAVID Bioinformatics Tool version 6.8 (david.ncifcrf.gov/). Enrichment analysis was performed using the functional annotation chart and was limited to GO terms in the 'Biological Process direct' and KEGG pathways categories. Functional annotations with a $\mathrm{P}<0.05$ and an enrichment score of $>2$ were considered significant. 
Statistical analysis. T-test for continuous variables and $\chi^{2}$ test for categorical variables were performed to assess the relationship between the prognostic risk score and clinical features. In the $\chi^{2}$ analysis, when the total number of samples was $<40$ or the expected value of the sample as $<1$, Fisher exact test was used. Univariate and multivariate Cox regression analyses were performed to verify if the predictive indicators of prognostic risk score calculated from multiple lncRNAs were independent of other clinical features. Statistical analyses were performed using IBM SPSS statistics software program version 22.0 (IBM Corp., Armonk, NY, USA). P<0.05 was considered to indicate a statistically significant difference.

\section{Results}

Patient characteristic. In this study, expression profiles of 178 samples of pancreatic cancer tissue and 4 samples of normal tissue were used to identify DElncRNAs. These samples were from 177 patients with pancreatic cancer included the following clinical and pathological characteristics: Age, sex, tumor site, tumor size, grade, stage, TNM status, chronic pancreatitis, smoking, alcohol consumption and diabetes (Table I).

DElncRNAs between pancreatic tumor tissues and normal tissues. According to the screening criteria $(\mathrm{P}<0.05$ and $\mid \log _{2} \mathrm{FCl}>2.0$ ), a total of 92 DElncRNAs were identified between pancreatic tumor tissues and normal tissues, including 3 (3.3\%) upregulated and 89 (96.7\%) downregulated lncRNAs. In order to visually display DElncRNA between the two groups, the results were presented as a volcano plot (Fig. 1). Unsupervised hierarchical cluster analysis showed that normal tissues could be distinguished from pancreatic cancer tissues based on the DElncRNA patterns (Fig. 2).

Identification of potential lncRNA biomarkers significantly associated with OS from DElncRNAs. Kaplan-Meier curves and the log-rank method were used to identify potential IncRNA biomarkers significantly associated with OS from the DElncRNAs. The results showed that seven lncRNAs [AC008033.3, AC079015.1, MIR600 host gene (MIR600HG), AP003086.1, FAM53B antisense RNA 1 (FAM53B-AS1), DNAH17 antisense RNA 1 (DNAH17-AS1), AL137789.1; Table II] were significantly associated with pancreatic cancer prognosis. Of these, five were positively associated with OS (AC008033.3, AC079015.1, MIR600HG, AP003086.1 and FAM53B-AS1; Fig. 3A-E), and two were negatively associated with OS (DNAH17-AS1 and AL137789.1, Fig. 3F and G).

Establishment of a three-lncRNA signature associated with pancreatic cancer patient survival. Three lncRNAs (MIR600HG, AL137789.1, AC079015.1) were selected from seven potential prognostic lncRNAs for logistic regression analysis according to the algorithm by trying to incorporate fewer IncRNAs, while achieving the best fit for patient's survival. Then, a formula was established using these three-lncRNAs to assess prognostic risk as follows: Risk score $=(0.3073 \mathrm{x}$ expression value of AL137789.1) + (-0.2044 x expression value of AC079015.1) + (-0.7195 x expression value of MIR600HG) The risk score for each patient was calculated and all patients were divided into a high-risk group (88 cases) and
Table I. Patient demographics and clinicopathological factors.

Characteristic Number of patients

Age (years)

$<60 / \geq 60$

$54 / 123$

Sex

Male/female

Tumor site

Head/other/NA

$138 / 28 / 11$

Tumor size

$<4 / \geq 4 \mathrm{~cm} / \mathrm{NA}$

$96 / 68 / 13$

Grade

$1 / 2 / 3 / 4 / x$

Stage

I/IIA/IIB/III/IV

$21 / 28 / 117 / 3 / 4$

$\mathrm{T}$

T1/T2/T3/T4/Tx

$8 / 24 / 141 / 3 / 1$

$\mathrm{N}$

$\mathrm{N} 0 / \mathrm{N} 1 / \mathrm{Nx}$

$49 / 123 / 5$

$\mathrm{M}$

$\mathrm{M} 0 / \mathrm{M} 1 / \mathrm{Mx}$

79/4/94

Chronic pancreatitis

None/yes/NA

Smoking (years)

$<3 / \geq 3 /$ NA

$84 / 58 / 35$

Drinking

None/yes/NA

$64 / 101 / 12$

Diabetes

None/yes/NA

$108 / 37 / 32$

NA, not available; $\mathrm{T}$, tumor; $\mathrm{N}$, node; $\mathrm{M}$, metastasis.

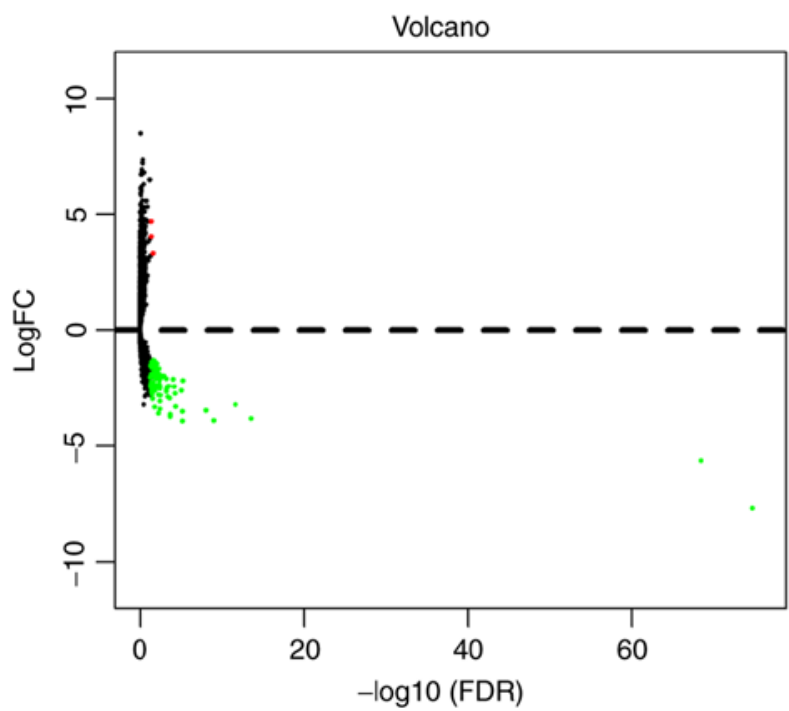

Figure 1. Volcano plot of differentially expressed lncRNAs. Red dots represent upregulated lncRNAs and green dots represent downregulated lncRNAs. lncRNA, long non-coding RNA; FC, fold change; FDR, false discovery rate. 


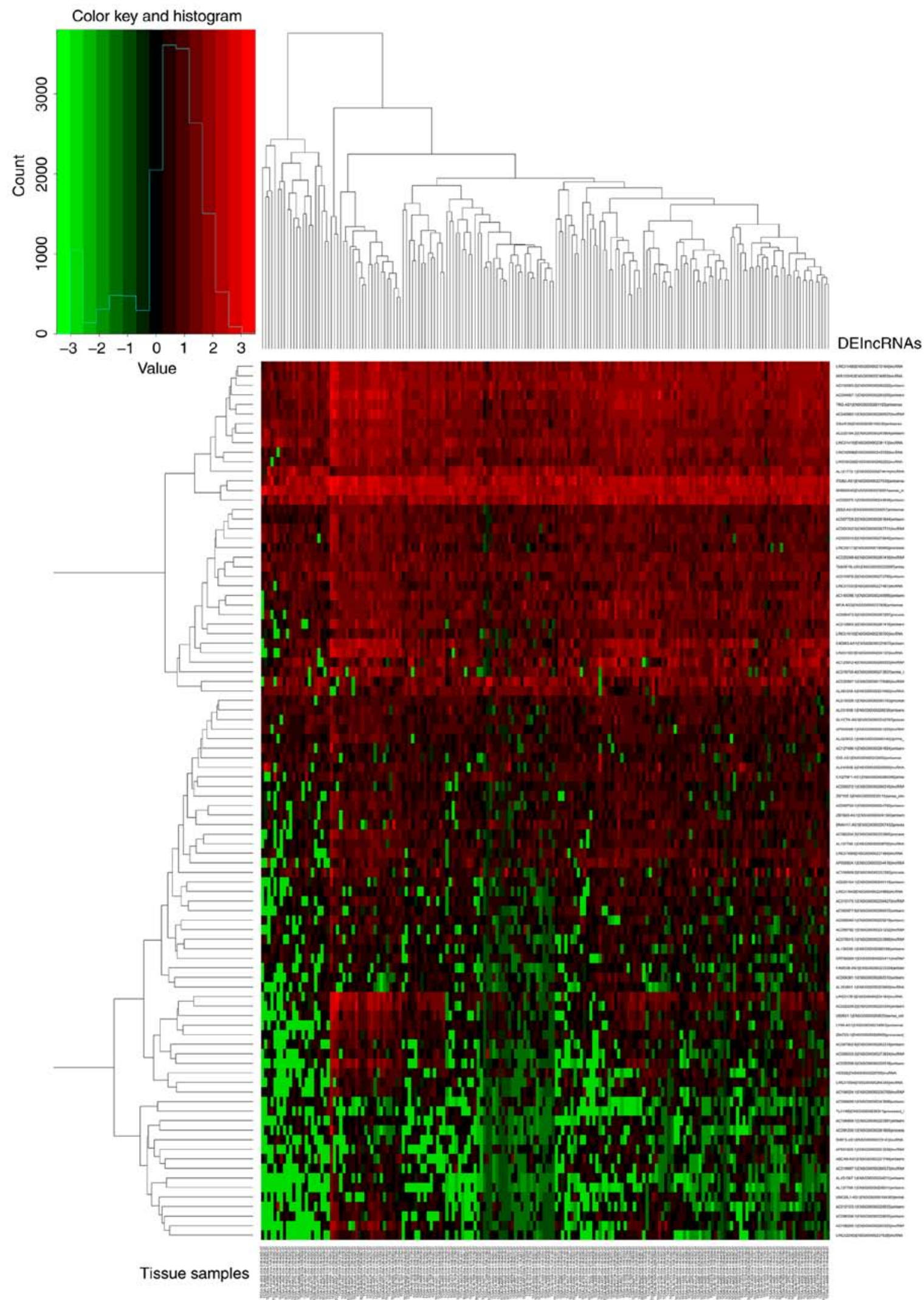

Figure 2. Unsupervised hierarchic cluster analysis revealed that pancreatic cancer tissues could be distinguished from normal tissues based on DElncRNA patterns. DElncRNA, differentially expressed long non-coding RNA.

a low-risk group (89 cases) according to the cut-off median risk score (Fig. 4A and B). The OS of the high-risk group was significantly shorter than that of the low-risk group (median OS 1.33 vs. 3.65 years; $\mathrm{P}<0.0001)$. The Kaplan-Meier survival curve is shown in Fig. 4C. As the risk score rose, the expression of AL137789.1 increased, and the expression of MIR600HG and AC079015.1 decreased (Fig. 4D). To evaluate the ability of the risk score to predict 3- and 5-year survival rates, a 
Table II. Seven long non-coding RNAs significantly associated with the overall survival of patients with pancreatic cancer.

\begin{tabular}{llcllll} 
Gene symbol & \multicolumn{1}{c}{ Ensembl ID } & \multicolumn{1}{c}{ Chromosome $(G R C h 38)$} & Log fold change & P-value & $\begin{array}{c}\text { False } \\
\text { discovery rate }\end{array}$ & $\begin{array}{l}\text { Adjusted } \\
\text { P-value }\end{array}$ \\
\hline DNAH17-AS1 & ENSG00000267432 & $17: 78,484,882-78,503,056$ & -3.46188237 & $6.46871 \mathrm{E}-12$ & $8.72 \mathrm{E}-09$ & 0.00565 \\
AL137789.1 & ENSG00000236911 & $1: 207,551,925-207,606,555$ & -3.62282343 & $3.81106 \mathrm{E}-07$ & 0.000198 & 0.03455 \\
AC079015.1 & ENSG00000253988 & $8: 138,063,268-138,073,240$ & -2.46900918 & $1.08231 \mathrm{E}-06$ & 0.000438 & 0.01524 \\
FAM53B-AS1 & ENSG00000233334 & $10: 124,703,625-124,714,217$ & -2.07318081 & $2.6809 \mathrm{E}-05$ & 0.00529 & 0.0453 \\
AP003086.1 & ENSG00000251323 & $11: 78,423,982-78,429,836$ & -1.73351271 & $7.38534 \mathrm{E}-05$ & 0.012111 & 0.00115 \\
MIR600HG & ENSG00000236901 & $9: 123,109,494-123,115,477$ & -1.60319345 & $9.46888 \mathrm{E}-05$ & 0.013928 & 0.00736 \\
AC008033.3 & ENSG00000273824 & $12: 68,426,331-68,427,737$ & -2.5734859 & 0.000352377 & 0.035634 & 0.00959
\end{tabular}

DNAH17-AS1, DNAH17 antisense RNA 1; FAM53B-AS1, FAM53B antisense RNA 1; MIR600HG, MIR600 host gene.

A
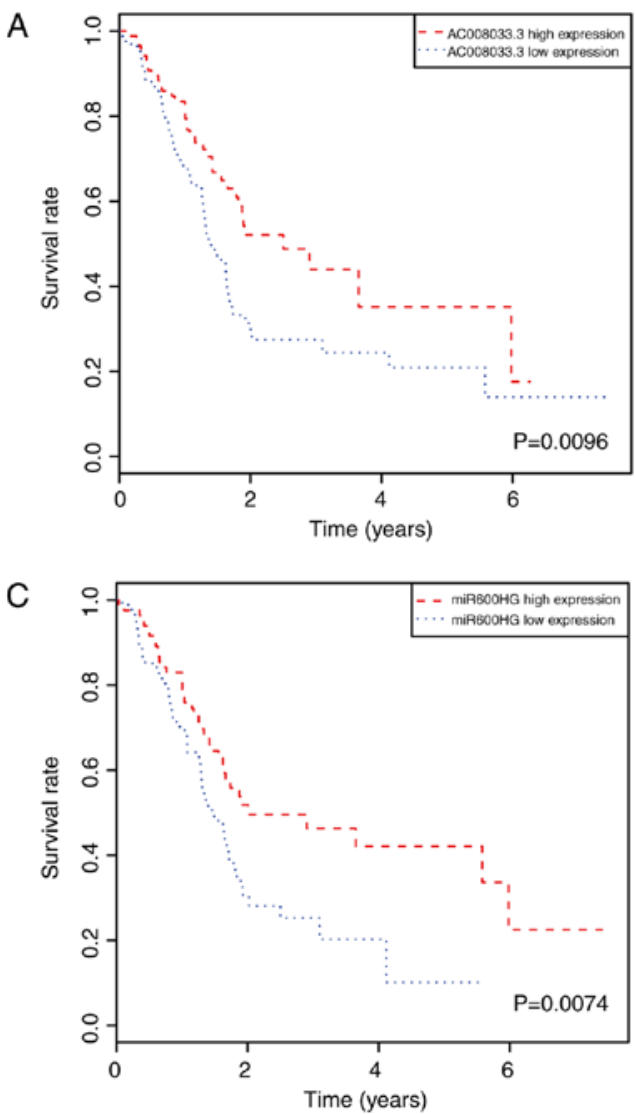

B

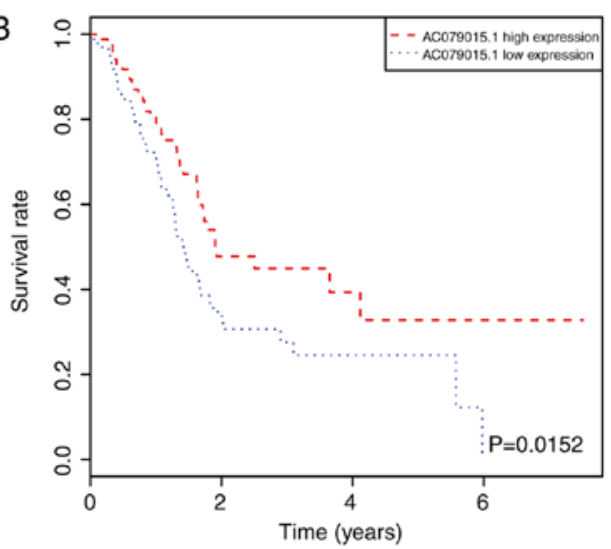

$\mathrm{D}$

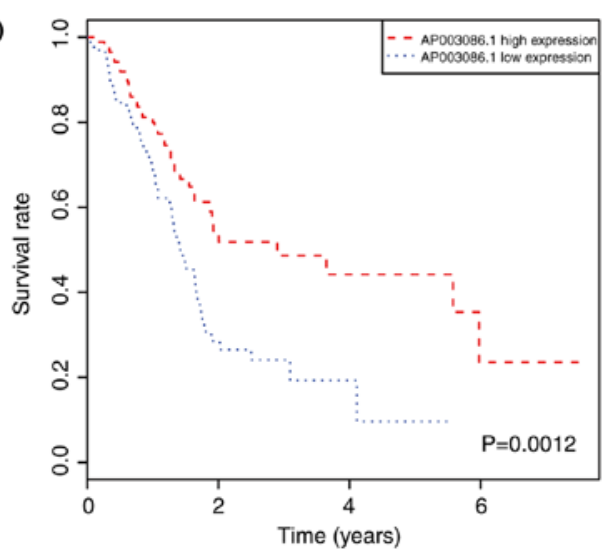

Figure 3. Kaplan-Meier estimates of overall survival of patients with pancreatic cancer from The Cancer Genome Atlas based on single long non-coding RNAs. (A) AC008033.3, (B) AC079015.1, (C) MIR600HG, (D) AP003086.1.

time-dependent ROC curve was used (Fig. 5). The results showed that the AUC for the lncRNA signature prognostic model was 0.742 for an OS of 3 years and 0.793 for an OS of 5 years. These results suggested an improvement in survival predictions based on the expression of these three pancreatic cancer lncRNAs.

Association between the three-lncRNA signature risk score and clinicopathological characteristics. Significant differences in tumor size, American Joint Committee on Cancer (AJCC) (22) stage, AJCC tumor status (T) and AJCC node status $(\mathrm{N})$ were identified between patients in with high and low risk scores; however, there was no difference in age, sex, tumor site, grade, AJCC metastasis status, chronic pancreatitis or smoking between the patients with high and low risk scores (Table III).

Prognostic value of the three-lncRNA signature is independent of other clinicopathological factors. Furthermore, univariate analyses were used to test the prognostic value of the three-lncRNA signature on OS. The results showed that risk score [hazard ratio $(\mathrm{HR})=2.442 ; \mathrm{P}<0.001)$, tumor site 

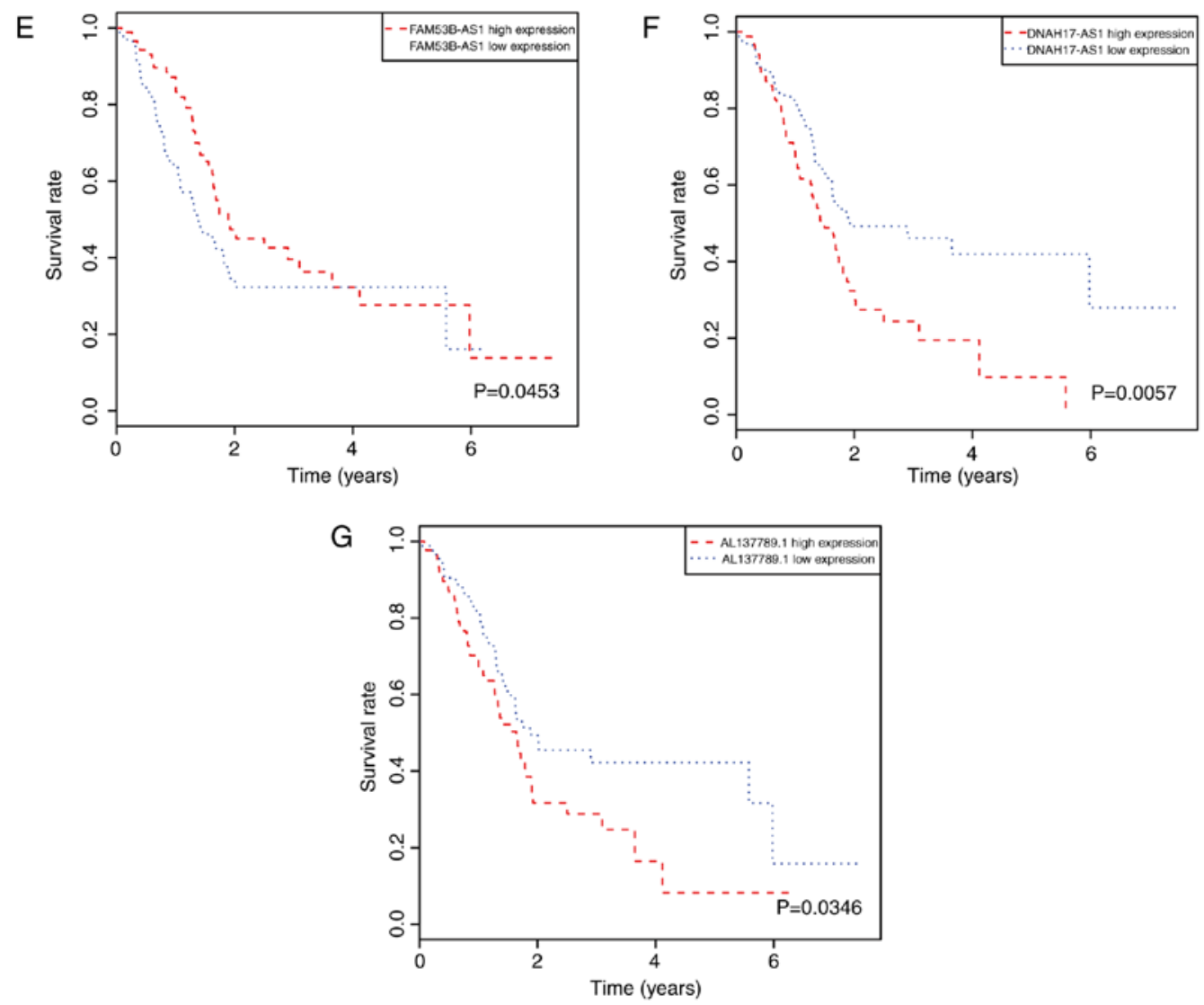

Figure 3. Continued. (E) FAM53B-AS1, (F) DNAH17-AS1, (G) AL137789.1. MIR600HG, MIR600 host gene; FAM53B-AS1, FAM53B antisense RNA 1; DNAH17-AS1, DNAH17 antisense RNA 1.

( $\mathrm{HR}=0.343 ; \mathrm{P}=0.004)$, grade $(\mathrm{HR}=1.584 ; \mathrm{P}=0.035)$, AJCC stage $(\mathrm{HR}=2.295: \mathrm{P}=0.001)$, AJCC T $(\mathrm{HR}=1.774 ; \mathrm{P}=0.051)$, and AJCC N (HR=1.968; P=0.008) were associated with OS in patients with pancreatic cancer (Table IV). These significant indicators from univariate analyses were subsequently included in multivariate Cox regression analyses (because AJCC stage is not independent of the AJCC T and AJCC N indicators, AJCC stage was excluded as an indicator). The results of multivariate Cox regression analyses indicated that risk score $(\mathrm{HR}=1.909 ; \mathrm{P}=0.007)$ is an independent prognostic factor for pancreatic cancer and that a high risk score is associated with a poor prognosis (Table IV).

Establishment of an lncRNA-mRNA co-expression network and lncRNA functional predictions. To investigate the potential biological functions of the DElncRNAs, a co-expression network was established between DElncRNAs and DEprotein-coding genes (Fig. 6A). The expression of 153 protein-coding genes from 1,452 DEmRNAs was highly correlated with 18 DElncRNAs (Pearson correlation coefficient $>0.60$ ). IncRNA AL137789.1, which is part of the three-lncRNA signature, was also in this co-expression network. Subsequently, the co-expressed protein-encoding genes were subjected to GO and KEGG pathway enrichment analyses, with the background of the whole human genome. GO functional annotation suggested that 153 protein-coding genes were significantly enriched in $63 \mathrm{GO}$ terms. The top $20 \mathrm{GO}$ terms are presented in Fig. 6B. KEGG functional annotation suggested that protein-coding genes were significantly enriched in 40 KEGG pathways. The top 20 pathways are presented in Fig. 6C. The results from GO and KEGG analyses were associated with immune responses involving $\mathrm{T}, \mathrm{B}$ and natural killer cells. Taken together, these results suggested that lncRNAs may be involved in the tumor immune response via interactions with protein-coding genes.

\section{Discussion}

The traditional view suggests that non-coding RNAs do not affect gene expression. However, these non-coding RNAs regulate the expression of genes at different levels, and it has been reported that they can even encode proteins involved in biological processes (23). Increasing numbers of abnormally expressed lncRNAs have been identified in a variety of tumors (6). These IncRNAs have complex biological functions, including tumor promotion and tumor suppression, which may be closely associated tumorigenesis and tumor progression.

It has been reported that the IncRNAs HOXA11 antisense RNA (24), DiGeorge syndrome critical region gene 5 (25), maternally expressed 3 (26) and hepatocellular carcinoma upregulated long non-coding RNA (27) promote metastasis and tumor cell proliferation and are associated with poor prognosis in cancer patients. However, the lncRNAs CPS1 intronic transcript 1 (28), cancer susceptibility 2 (29), long intergenic non-protein coding RNA 589 (30) and growth arrest specific 5 (31) can inhibit the proliferation and metastasis of 
Table III. Association between the risk score of three-long noncoding RNAs signature and clinicopathological characteristics.

\begin{tabular}{|c|c|c|c|c|}
\hline \multirow[b]{2}{*}{ Characteristic } & \multicolumn{2}{|c|}{$\begin{array}{c}\text { Number } \\
\text { of patients }(\%)\end{array}$} & \multirow[b]{2}{*}{$\chi^{2}$} & \multirow[b]{2}{*}{ P-value } \\
\hline & Low risk & High risk & & \\
\hline Total patients & 89 (100) & $88(100)$ & & \\
\hline Age (years) & & & 0.077 & 0.782 \\
\hline$<60$ & $28(31.5)$ & $26(29.5)$ & & \\
\hline$\geq 60$ & $61(68.5)$ & $62(70.5)$ & & \\
\hline Sex & & & 0.950 & 0.330 \\
\hline Male & $52(58.4 \%)$ & $45(51.1 \%)$ & & \\
\hline Female & $37(41.6 \%)$ & $43(48.9 \%)$ & & \\
\hline Tumor site & & & 0.020 & 0.889 \\
\hline Head & $67(75.3)$ & $71(80.7)$ & & \\
\hline Others & $14(15.7)$ & $14(15.9)$ & & \\
\hline NA & $8(9.0)$ & $3(3.4)$ & & \\
\hline Tumor size $(\mathrm{cm})$ & & & 5.796 & 0.016 \\
\hline$<4$ & $54(60.7)$ & $42(47.7)$ & & \\
\hline$\geq 4$ & $27(30.3)$ & $41(46.6)$ & & \\
\hline NA & $8(9.0 \%)$ & $5(5.7 \%)$ & & \\
\hline Grade & & & 0.005 & 0.945 \\
\hline $\mathrm{G}_{1}+\mathrm{G}_{2}$ & $62(69.7)$ & $61(69.3)$ & & \\
\hline $\mathrm{G}_{3}+\mathrm{G}_{4}$ & $26(29.2)$ & $25(28.4)$ & & \\
\hline $\mathrm{G}_{\mathrm{X}}$ & $1(1.1)$ & $2(2.3)$ & & \\
\hline AJCC stage & & & 4.192 & 0.041 \\
\hline $\mathrm{I}+\mathrm{IIA}$ & $31(34.8)$ & $18(20.5)$ & & \\
\hline $\mathrm{IIB}+\mathrm{III}+\mathrm{IV}$ & $56(62.9)$ & $68(77.3)$ & & \\
\hline NA & $2(2.2)$ & $2(2.3)$ & & \\
\hline AJCC T & & & 6.116 & 0.013 \\
\hline $\mathrm{T}_{1}+\mathrm{T}_{2}$ & $22(24.7)$ & $10(11.4)$ & & \\
\hline $\mathrm{T}_{3}+\mathrm{T}_{4}$ & $66(74.1)$ & $78(88.7)$ & & \\
\hline $\mathrm{T}_{\mathrm{X}}$ & $1(1.1)$ & $0(0.0)$ & & \\
\hline AJCC N & & & 5.256 & 0.022 \\
\hline $\mathrm{N}_{0}$ & $31(34.8)$ & $18(20.5)$ & & \\
\hline $\mathrm{N}_{1}$ & $54(60.7)$ & $69(78.4)$ & & \\
\hline $\mathrm{N}_{\mathrm{X}}$ & $4(4.5)$ & $1(1.1)$ & & \\
\hline AJCC M & & & & 0.348 \\
\hline $\mathrm{M}_{0}$ & $42(47.2)$ & $37(42.0)$ & & \\
\hline $\mathrm{M}_{1}$ & $1(1.1)$ & $3(3.4)$ & & \\
\hline $\mathrm{M}_{\mathrm{X}}$ & $46(51.7)$ & $48(54.5)$ & & \\
\hline Chronic pancreatitis & & & 0.013 & 0.910 \\
\hline None & $65(73.0)$ & $61(69.3)$ & & \\
\hline Yes & $7(7.9)$ & $7(8.0)$ & & \\
\hline NA & $17(19.1)$ & $20(22.7)$ & & \\
\hline Smoking (years) & & & 1.049 & 0.306 \\
\hline$<3$ & $45(50.6)$ & $39(44.3)$ & & \\
\hline$\geq 3$ & $26(29.2)$ & $32(36.4)$ & & \\
\hline NA & $18(20.3)$ & $17(19.3)$ & & \\
\hline Alcohol consumption & & & 0.004 & 0.951 \\
\hline None & $32(36)$ & $32(36.4)$ & & \\
\hline Yes & $51(57.3)$ & $50(56.8)$ & & \\
\hline NA & $6(6.7)$ & $6(6.8)$ & & \\
\hline
\end{tabular}

Table III. Continued.

\begin{tabular}{lllll}
\hline & \multicolumn{2}{c}{$\begin{array}{c}\text { Number } \\
\text { of patients }(\%)\end{array}$} & & \\
\cline { 2 - 3 } Characteristic & Low risk & High risk & $\chi^{2}$ & P-value \\
\hline Diabetes & & & 1.651 & 0.199 \\
None & $51(57.3)$ & $57(64.8)$ & & \\
Yes & $22(24.7)$ & $15(17.0)$ & & \\
NA & $16(18.0)$ & $16(18.2)$ & & \\
\hline
\end{tabular}

NA, not available; AJCC, American Joint Committee on Cancer; $\mathrm{T}$, tumor; $\mathrm{N}$, node; $\mathrm{M}$, metastasis.
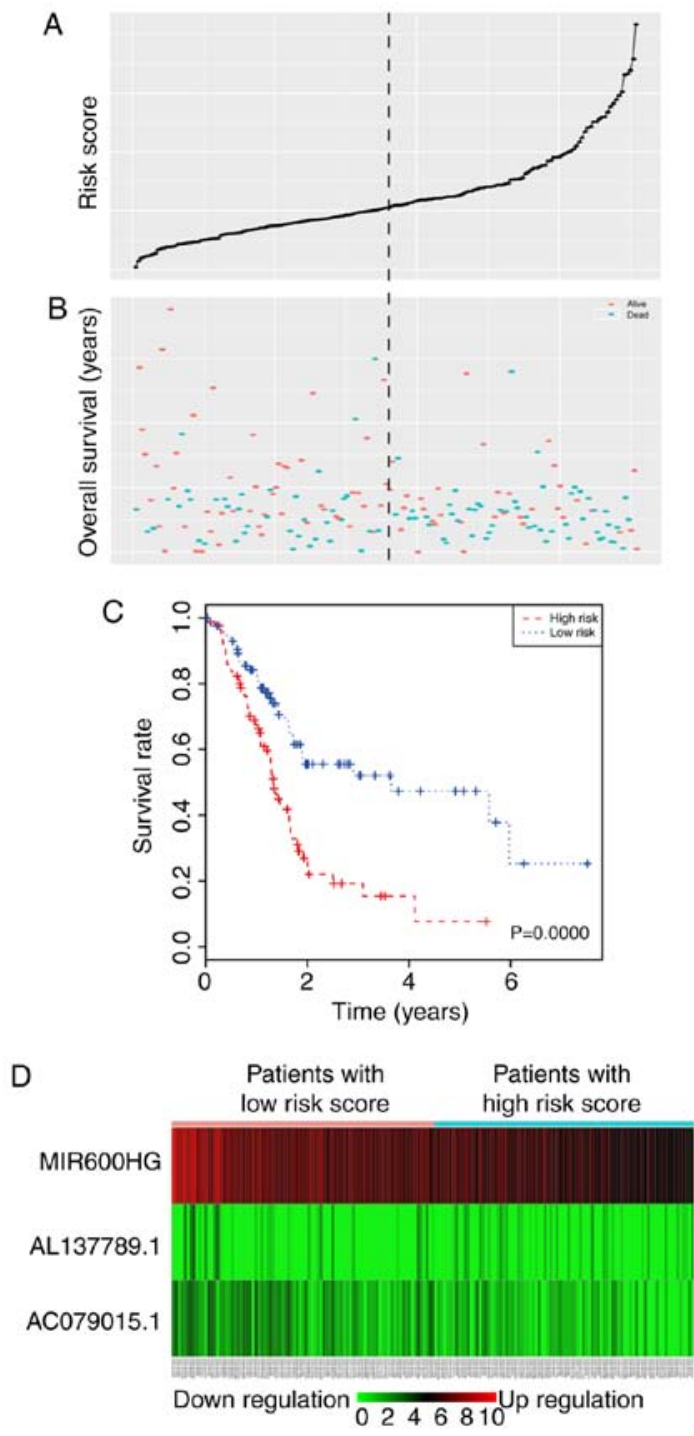

Figure 4. Three-lncRNA risk score analysis of the TCGA patients. The distribution of the three-lncRNA risk score, overall survival status and IncRNA expression signature were analyzed in the entire TCGA patient cohort (n=177). (A) Three-lncRNA risk score distribution; (B) patient overall survival status and survival length. The dotted line divides the patients into low-risk and high-risk groups. Patients were divided into two groups based on median risk score. (C) Kaplan-Meier curves for low- and high-risk patients with pancreatic cancer. (D) Risk heatmap of the three-lncRNA expression profiles. As the risk score rose, the expression of AL137789.1 increased, and the expression of MIR600HG and AC079015.1 decreased. Rows represent lncRNAs in the signature, and columns represent patients. TCGA, The Cancer Genome Atlas; MIR600HG, MIR600 host gene. 
Table IV. Univariate and multivariate analyses of the three-long non-coding RNA signature in the prediction of pancreatic cancer overall survival.

\begin{tabular}{|c|c|c|c|c|}
\hline & \multicolumn{2}{|c|}{ Univariate analysis } & \multicolumn{2}{|c|}{ Multivariate analysis } \\
\hline & $\mathrm{HR}(95 \% \mathrm{CI})$ & P-value & $\mathrm{HR}(95 \% \mathrm{CI})$ & P-value \\
\hline Risk score (high vs. low) & $2.442(1.576-3.784)$ & 0.000 & $1.909(1.195-3.050)$ & 0.007 \\
\hline Age, years $(<60$ vs. $<60)$ & $1.291(0.820-2.035)$ & 0.270 & & \\
\hline Sex (female vs. male) & $0.900(0.597-1.357)$ & 0.616 & & \\
\hline Tumor site (other vs. head) & $0.343(0.165-0.712)$ & 0.004 & $0.415(0.194-0.885)$ & 0.023 \\
\hline Tumor Size ( $\geq 4 \mathrm{~cm}$ vs. $<4 \mathrm{~cm}$ ) & $1.016(0.666-1.549)$ & 0.943 & & \\
\hline Grade $\left(G_{3}+G_{4}\right.$ vs. $\left.G_{1}+G_{2}\right)$ & $1.584(1.032-2.432)$ & 0.035 & $1.643(1.052-2.567)$ & 0.029 \\
\hline AJCC stage (IIB + III + IV vs. I+IIA) & $2.295(1.383-3.808)$ & 0.001 & & \\
\hline $\operatorname{AJCC~T~}\left(\mathrm{T}_{3}+\mathrm{T}_{4}\right.$ vs. $\left.\mathrm{T}_{1}+\mathrm{T}_{2}\right)$ & $1.774(0.997-3.159)$ & 0.051 & $0.937(0.496-1.769)$ & 0.841 \\
\hline $\operatorname{AJCC~N}\left(\mathrm{N}_{1}\right.$ vs. $\left.\mathrm{N}_{0}\right)$ & $1.968(1.191-3.252)$ & 0.008 & $1.596(0.877-2.904)$ & 0.126 \\
\hline $\operatorname{AJCC} \mathrm{M}\left(\mathrm{M}_{1}\right.$ vs. $\left.\mathrm{M}_{0}\right)$ & $0.571(0.078-4.192)$ & 0.581 & & \\
\hline Chronic pancreatitis (yes vs. none) & $1.008(0.460-2.208)$ & 0.983 & & \\
\hline Smoking ( 3 years vs. $<3$ years) & $0.941(0.595-1.490)$ & 0.797 & & \\
\hline Alcohol consumption (yes vs. none) & $1.249(0.805-3.159)$ & 0.322 & & \\
\hline Diabetes (yes vs. none) & $0.902(0.518-1.569)$ & 0.714 & & \\
\hline
\end{tabular}

HR, hazard ratio; CI, confidence interval; AJCC, American Joint Committee on Cancer; T, tumor; N, node; M, metastasis.

A

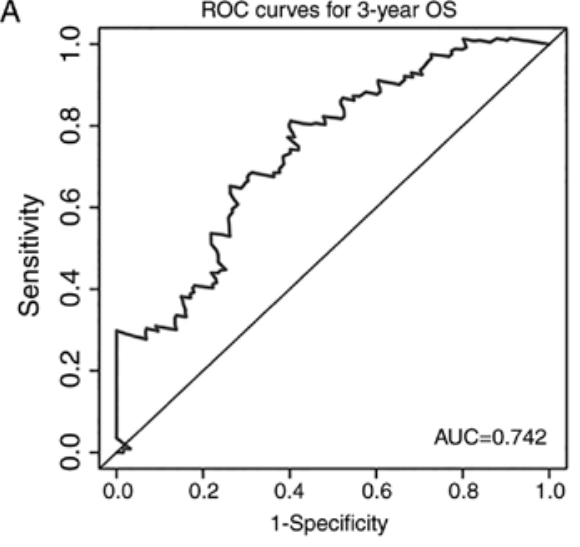

B

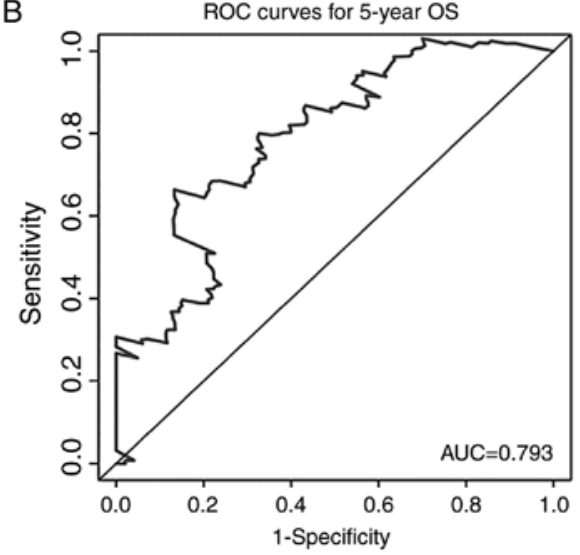

Figure 5. ROC curves of the multivariate logistic regression model with or without risk score using the three-lncRNA signature to predict (A) 3-year and (B) 5-year survival. ROC, receiver operating characteristic; OS, overall survival; AUC, area under the curve.

tumor cells, which indicates a favorable survival. The role of certain lncRNAs, such as taurine upregulated 1 (TUG1), in tumors is controversial. Studies have reported that TUG1 contributes to tumor progression (32-34) via its role in the promotion of cell proliferation, metastasis and inhibition of cell apoptosis. Thus, it is thought to act as an oncogene in various cancers, such as ovarian (35), thyroid (34) and colorectal cancer (36). However, other studies have reported that the lncRNA TUG1 is a tumor suppressor $(37,38)$. A similar controversy occurred for the lncRNA prostate cancer associated 3 (PCA3) $(39,40)$. However, ncRNA accounts for $80 \%$ of the whole genome, and the general understanding of the function of lncRNAs is still superficial. Many lncRNAs have not been functionally investigated.
In pancreatic cancer, several single lncRNA biomarkers have been reported, including lncRNA PVT1 oncogene (41), CCDC26 lncRNA (42) and long intergenic non-protein coding RNA, p53 induced transcript (43). The same controversy that occurred around PCA3 in prostate cancer may also occur in pancreatic cancer. The expression of lncRNAs is relatively low, thus it may be easy to introduce bias when using a single lncRNA as a biomarker. Utilizing a combination of multiple potential lncRNA biomarkers could improve accuracy. In this study, a three-lncRNA signature that is associated with OS was established by analyzing the RNA-sequencing data from 178 patients with pancreatic cancer from the TCGA database.

The lncRNAs creating the three-lncRNA signature were MIR600HG, AL137789.1 and AC079015.1. To the best of our 
A

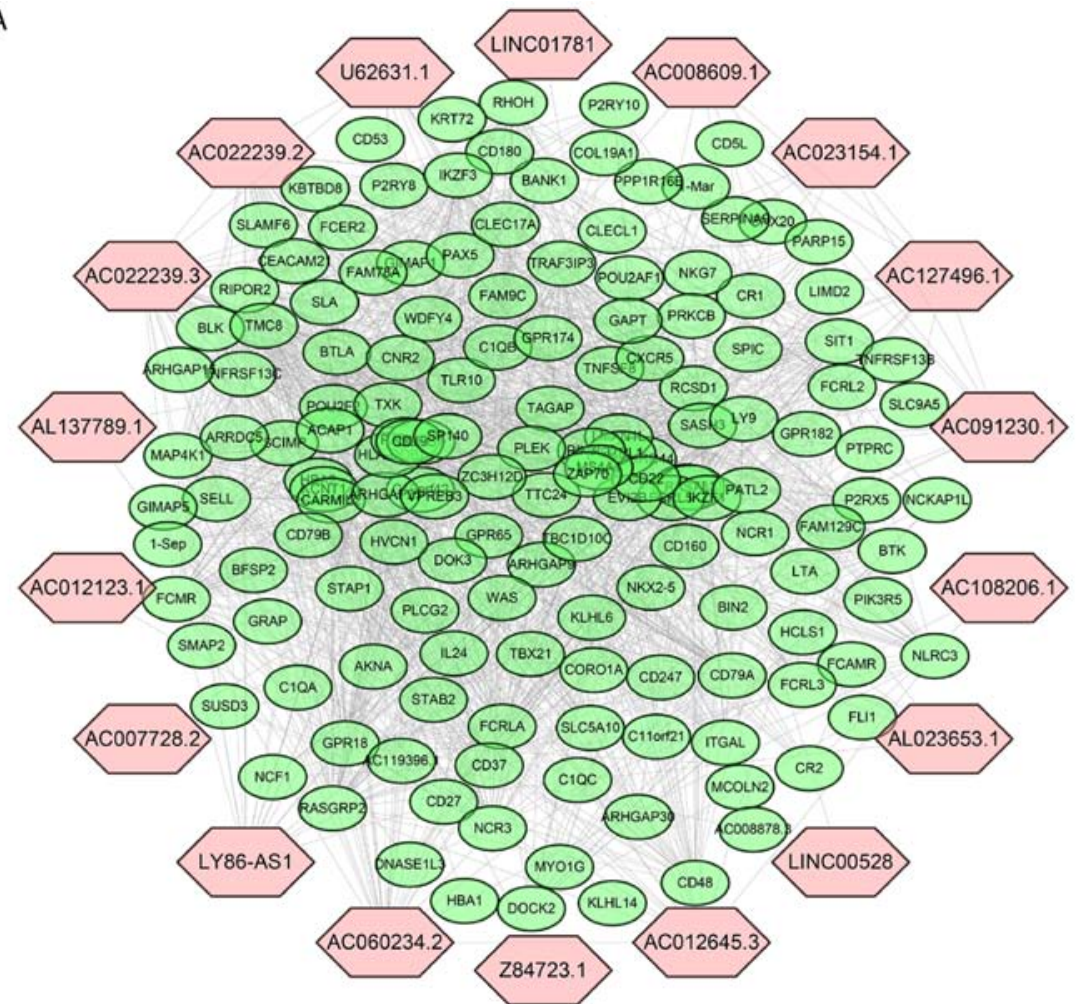

B

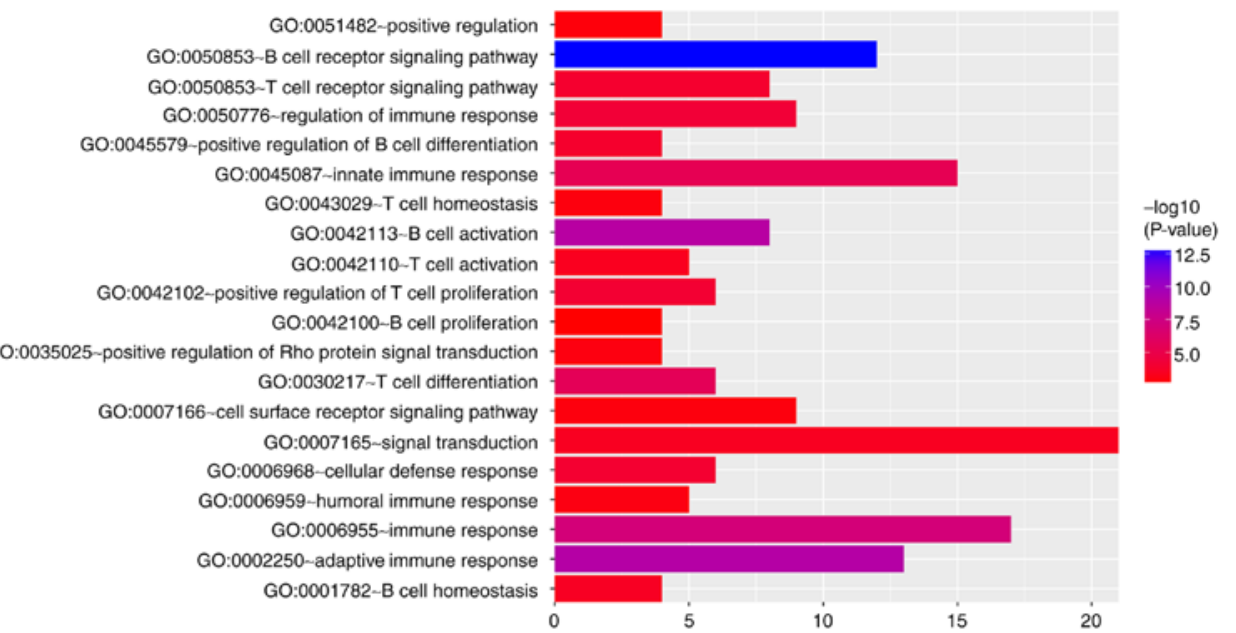

C

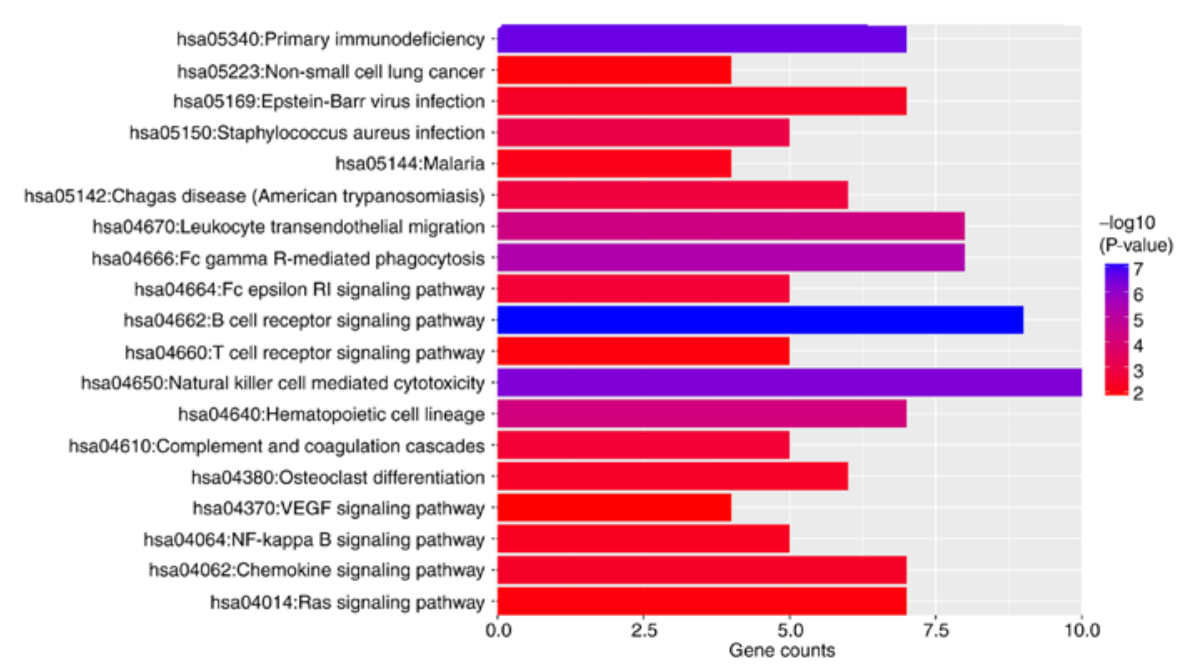

Figure 6. Co-expression network and functional enrichment results. (A) Co-expression network between DElncRNAs and DEprotein-coding genes. IncRNAs, pink hexagons; mRNAs, green ovals. (B) Top 20 significantly enriched GO terms from the co-expressed protein-coding genes and DElncRNAs. (C) Top 20 significantly enriched Kyoto Encyclopedia to Genes and Genomes pathways from the co-expressed protein-coding genes and DElncRNAs. DE, differentially expressed; lncRNA, long non-coding RNA; GO, Gene Ontology. 
knowledge, these three IncRNAs have not been previously reported in any cancer. The expression of all three lncRNAs was significantly lower in tumor tissues than in normal tissues. Further, the expression of these lncRNAs is closely associated with the OS of patients with pancreatic cancer. Lower expression of AC079015.1 and MIR600HG was associated with poorer OS, whereas lower expression of IncRNA AL137789.1 was associated with favorable survival. A calculation for prognosis risk score in patients with pancreatic cancer was established according to the expression of the three lncRNAs. The formula indicates that a higher risk score is associated with a poorer prognosis. Multivariate analyses demonstrated that the three-lncRNA signature was an independent prognostic predictor of survival for patients with pancreatic cancer patient. Further, ROC analyses showed that the AUC of the three-lncRNA signature was 0.742 for the prediction of 3-year OS and 0.793 for the prediction of 5-year OS. These results demonstrate that these three IncRNAs have a high accuracy in predicting the prognosis of patients with pancreatic cancer. In analyses of the association between risk score and clinicopathological characteristics, high risk scores were significantly associated with larger tumor size and higher TNM stage. This indicated that high risk is associated with tumor progression from another perspective.

Thus far, the function of these three IncRNAs is unknown. A lncRNA-mRNA co-expression network was used to predict the function of lncRNAs by functional analysis of protein-coding genes. The GO and KEGG enrichment results implied that the DElncRNAs may be involved in tumorigenesis through effects on the tumor immune response. Unfortunately, AL137789.1 was the only lncRNA from three-lncRNA signature that was involved in the co-expression network. AL137789.1 may increase the malignancy of pancreatic cancer by affecting the tumor immune microenvironment, despite the current lack of supporting evidence. A large number of studies have demonstrated that tumor biological behavior is regulated by the tumor immune microenvironment. In pancreatic cancer, the tumor immune microenvironment is more complicated due to the rich stromal environment of the pancreas. Tumor-infiltrating lymphocytes and cytokines secreted from the stroma constitute the main components of the tumor immune microenvironment, and have a role in the immune regulation of tumors. However, lncRNAs may be involved in certain tumor immunity processes and may aid in tumor cell immune evasion $(44,45)$. Notably, studies have reported that lncRNAs are involved in the human immune system, including roles in dendritic cells, $\mathrm{T}$ cells and macrophages $(44,45)$. Jiang et al $(45)$ reported that the lncRNA lnc-epidermal growth factor receptor stimulates T-regulatory cell differentiation, thus promoting hepatocellular carcinoma immune evasion. Another study reported that the IncRNA IFNG antisense RNA 1, which is expressed by Th1 cells, specifically decreases the differentiation of T-cells toward the Th2 phenotype (46).

In summary, the current study established a three-lncRNA signature associated with pancreatic cancer by examining lncRNA expression profiles from the TCGA database. Further analysis demonstrated that the three-lncRNA signature may be an independent prognostic biomarker of survival of patients with pancreatic cancer. Functional predictions revealed that one of these three lncRNAs, AL137789.1, may be associated with the tumor immune response.

\section{Acknowledgements}

Not applicable.

\section{Funding}

The present study was supported in part by the Public Welfare Technology Research and Social Development Project of the Department of Science and Technology of Zhejiang Province (No. 2014C33139) and the Social Development Research and Demonstration Application Project of the Bureau of Science and Technology of Jiaxing City (No. 2016AY23057).

\section{Availability of data and materials}

The analyzed data sets generated during the study are available from the corresponding author on reasonable request.

\section{Authors' contributions}

BW and $\mathrm{ZZ}$ designed the experiments and analyzed the data; YB and XW analyzed the data; KW, FC and JG performed lncRNA functional predictions; JF and ZS performed survival analysis; all authors read and approved the manuscript and agree to be accountable for all aspects of the research in ensuring that the accuracy or integrity of any part of the work are appropriately investigated and resolved.

\section{Ethics approval and consent to participate}

Ethical approval was obtained from the Second Hospital of Jiaxing (Jiaxing, China).

\section{Patient consent for publication}

Not applicable.

\section{Competing interests}

The authors declare that they have no competing interests.

\section{References}

1. Kamisawa T, Wood LD, Itoi T and Takaori K: Pancreatic cancer. Lancet 388: 73-85, 2016.

2. Arkan MC: Cancer: Fat and the fate of pancreatic tumours Nature 536: 157-158, 2016.

3. Samuel N and Hudson TJ: The molecular and cellular heterogeneity of pancreatic ductal adenocarcinoma. Nat Rev Gastroenterol Hepatol 9: 77-87, 2011.

4. Birney E, Stamatoyannopoulos JA, Dutta A, Guigó R, Gingeras TR, Margulies EH, Weng Z, Snyder M, Dermitzakis ET, Thurman RE, et al: Identification and analysis of functional elements in $1 \%$ of the human genome by the ENCODE pilot project. Nature 447: 799-816, 2007.

5. Lander ES, Linton LM, Birren B, Nusbaum C, Zody MC, Baldwin J, Devon K, Dewar K, Doyle M, FitzHugh W, et al: Initial sequencing and analysis of the human genome. Nature 409: 860-921, 2001.

6. Fatica A and Bozzoni I: Long non-coding RNAs: New players in cell differentiation and development. Nat Rev Genet 15: 7-21, 2014.

7. Chen Z, Chen X, Chen P, Yu S, Nie F, Lu B, Zhang T, Zhou Y, Chen $\mathrm{Q}$, Wei C, et al: Long non-coding RNA SNHG20 promotes non-small cell lung cancer cell proliferation and migration by epigenetically silencing of P21 expression. Cell Death Dis 8: e3092, 2017. 
8. Lin J, Tan X, Qiu L, Huang L, Zhou Y, Pan Z, Liu R, Chen S, Geng R, Wu J and Huang W: Long noncoding RNA BC032913 as a novel therapeutic target for colorectal cancer that suppresses metastasis by upregulating TIMP3. Mol Ther Nucleic Acids 8: 469-481, 2017

9. Tay Y, Rinn J and Pandolfi PP: The multilayered complexity of ceRNA crosstalk and competition. Nature 505: 344-352, 2014.

10. Yu Y, Nangia-Makker P, Farhana L and Majumdar APN: A novel mechanism of IncRNA and miRNA interaction: CCAT2 regulates miR-145 expression by suppressing its maturation process in colon cancer cells. Mol Cancer 16: 155, 2017.

11. Yan X, Zhang D, Wu W, Wu S, Qian J, Hao Y, Yan F, Zhu P, Wu J, Huang G, et al: Mesenchymal stem cells promote hepatocarcinogenesis via lncRNA-MUF interaction with ANXA2 and miR-34a. Cancer Res 77: 6704-6716, 2017.

12. Yang SZ, Xu F, Zhou T, Zhao X, McDonald JM and Chen Y: The long non-coding RNA HOTAIR enhances pancreatic cancer resistance to TNF-related apoptosis-inducing ligand. J Biol Chem 292: 10390-10397, 2017

13. Fu Z, Chen C, Zhou Q, Wang Y, Zhao Y, Zhao X, Li W, Zheng S, Ye H, Wang L, et al: LncRNA HOTTIP modulates cancer stem cell properties in human pancreatic cancer by regulating HOXA9. Cancer Lett 410: 68-81, 2017.

14. Liu JH, Chen G, Dang YW, Li CJ and Luo DZ: Expression and prognostic significance of IncRNA MALAT1 in pancreatic cancer tissues. Asian Pac J Cancer Prev 15: 2971-2977, 2014.

15. Li L, Chen H, Gao Y, Wang YW, Zhang GQ, Pan SH, Ji L, Kong R, Wang G, Jiia YH, et al: Long noncoding RNA MALAT1 promotes aggressive pancreatic cancer proliferation and metastasis via the stimulation of autophagy. Mol Cancer Ther 15: 2232-2243, 2016

16. Zhan HX, Wang Y, Li C, Xu JW, Zhou B, Zhu JK, Han HF, Wang L, Wang YS and Hu SY: LincRNA-ROR promotes invasion, metastasis and tumor growth in pancreatic cancer through activating ZEB1 pathway. Cancer Lett 374: 261-271, 2016.

17. Sun J, Cheng L, Shi H, Zhang Z, Zhao H, Wang Z and Zhou M: A potential panel of six-long non-coding RNA signature to improve survival prediction of diffuse large-B-cell lymphoma. Sci Rep 6: 27842,2016

18. Hu Y, Chen HY, Yu CY, Xu J, Wang JL, Qian J, Zhang X and Fang JY: A long non-coding RNA signature to improve prognosis prediction of colorectal cancer. Oncotarget 5: 2230-2242, 2014.

19. Li J, Chen Z, Tian L, Zhou C, He MY, Gao Y, Wang S, Zhou F, Shi S, Feng X, et al: LncRNA profile study reveals a three-lncRNA signature associated with the survival of patients with oesophageal squamous cell carcinoma. Gut 63: 1700-1710, 2014.

20. Zhou M, Xu W, Yue X, Zhao H, Wang Z, Shi H, Cheng L and Sun J: Relapse-related long non-coding RNA signature to improve prognosis prediction of lung adenocarcinoma. Oncotarget 7 : 29720-29738, 2016

21. Law CW, Alhamdoosh M, Su S, Smyth GK and Ritchie ME: RNA-seq analysis is easy as 1-2-3 with limma, Glimma and edgeR. F1000Res 5: 1408, 2016.

22. Cuccurullo V and Mansi L: AJCC cancer staging handbook: From the AJCC cancer staging manual (7th edition). Eur J Nucl Med Mol Imaging 38: 408-408, 2011.

23. Ponting CP, Oliver PL and Reik W: Evolution and functions of long noncoding RNAs. Cell 136: 629-641, 2009.

24. Liu Z, Chen Z, Fan R, Jiang B, Chen X, Chen Q, Nie F, Lu K and Sun M: Over-expressed long noncoding RNA HOXA11-AS promotes cell cycle progression and metastasis in gastric cancer Mol Cancer 16: 82, 2017.

25. Dong HX, Wang R, Jin XY, Zeng J and Pan J: LncRNA DGCR5 promotes lung adenocarcinoma (LUAD) progression via inhibiting hsa-mir-22-3p. J Cell Physiol 233: 4126-4136, 2018.

26. Gong $X$ and Huang M: Long non-coding RNA MEG3 promotes the proliferation of glioma cells through targeting Wnt/ $\beta$-catenin signal pathway. Cancer Gene Ther 24: 381-385, 2017.

27. Chen S, Wu DD, Sang XB, Wang LL, Zong ZH, Sun KX, Liu BL and Zhao Y: The lncRNA HULC functions as an oncogene by targeting ATG7 and ITGB1 in epithelial ovarian carcinoma. Cell Death Dis 8: e3118, 2017.

28. Zhang W, Yuan W, Song J, Wang S and Gu X: LncRNA CPS1-IT1 suppresses EMT and metastasis of colorectal cancer by inhibiting hypoxia-induced autophagy through inactivation of HIF-1 $\alpha$. Biochimie 144: 21-27, 2018.
29. Wang Y, Liu Z, Yao B, Li Q, Wang L, Wang C, Dou C, Xu M, Liu Q and Tu K: Long non-coding RNA CASC2 suppresses epithelial-mesenchymal transition of hepatocellular carcinoma cells through CASC2/miR-367/FBXW7 axis. Mol Cancer 16: 123,2017

30. Zhang J, Li Z, Liu L, Wang Q, Li S, Chen D, Hu Z, Yu T, Ding J, $\mathrm{Li}$ J, et al: Long noncoding RNA TSLNC8 is a tumor suppressor that inactivates the interleukin-6/STAT3 signaling pathway. Hepatology 67: 171-187, 2018.

31. Yang W, Hong L, Xu X, Wang Q, Huang J and Jiang L: LncRNA GAS5 suppresses the tumorigenesis of cervical cancer by downregulating miR-196a and miR-205. Tumour Biol 39: $1010428317711315,2017$.

32. Li Z, Shen J, Chan MT and Wu WK: TUG1: A pivotal oncogenic long non-coding RNA of human cancers. Cell Prolif 49: 471-475, 2016.

33. Liu L, Chen X, Zhang Y, Hu Y, Shen X and Zhu W: Long non-coding RNA TUG1 promotes endometrial cancer development via inhibiting miR-299 and miR-34a-5p. Oncotarget 8: 31386-31394, 2017.

34. Lei $\mathrm{H}, \mathrm{Gao} \mathrm{Y}$ and $\mathrm{Xu} \mathrm{X}$ : LncRNA TUG1 influences papillary thyroid cancer cell proliferation, migration and EMT formation through targeting miR-145. Acta Biochim Biophys Sin 49: 588-597, 2017.

35. Kuang D, Zhang X, Hua S, Dong W and Li Z: Long non-coding RNA TUG1 regulates ovarian cancer proliferation and metastasis via affecting epithelial-mesenchymal transition. Exp Mol Pathol 101: 267-273, 2016

36. Sun J, Ding C, Yang Z, Liu T, Zhang X, Zhao C and Wang J: The long non-coding RNA TUG1 indicates a poor prognosis for colorectal cancer and promotes metastasis by affecting epithelial-mesenchymal transition. J Transl Med 14: 42, 2016.

37. Zhang EB, Yin DD, Sun M, Kong R, Liu XH, You LH, Han L, Xia R, Wang KM, Yang JS, et al: P53-regulated long non-coding RNA TUG1 affects cell proliferation in human non-small cell lung cancer, partly through epigenetically regulating HOXB7 expression. Cell Death Dis 5: e1243, 2014.

38. Li J, Zhang M, An G and Ma Q: LncRNA TUG1 acts as a tumor suppressor in human glioma by promoting cell apoptosis. Exp Biol Med 241: 644-649, 2016.

39. Durand X, Xylinas E, Radulescu C, Haus-Cheymol R, Moutereau S, Ploussard G, Forgues A, Robert G, Vacherot F, Loric S, et al: The value of urinary prostate cancer gene 3 (PCA3) scores in predicting pathological features at radical prostatectomy. BJU Int 110: 43-49, 2012.

40. Augustin H, Mayrhofer K, Pummer K and Mannweiler S: Relationship between prostate cancer gene 3 (PCA3) and characteristics of tumor aggressiveness. Prostate 73: 203-210, 2013.

41. Xie Z, Chen X, Li J, Guo Y, Li H, Pan X, Jiang J, Liu H and Wu B: Salivary HOTAIR and PVT1 as novel biomarkers for early pancreatic cancer. Oncotarget 7: 25408-25419, 2016

42. Peng W and Jiang A: Long noncoding RNA CCDC26 as a potential predictor biomarker contributes to tumorigenesis in pancreatic cancer. Biomed Pharmacother 83: 712-717, 2016.

43. Li L, Zhang GQ, Chen H, Zhao ZJ, Chen HZ, Liu H, Wang G, Jia YH, Pan SH, Kong R, et al: Plasma and tumor levels of Linc-pint are diagnostic and prognostic biomarkers for pancreatic cancer. Oncotarget 7: 71773-71781, 2016.

44. Trimarchi T, Bilal E, Ntziachristos P, Fabbri G, Dalla-Favera R, Tsirigos A and Aifantis I: Genome-wide mapping and characterization of Notch-regulated long noncoding RNAs in acute leukemia. Cell 158: 593-606, 2014

45. Wang P, Xue Y, Han Y, Lin L, Wu C, Xu S, Jiang Z, Xu J, Liu Q and Cao X: The STAT3-binding long noncoding RNA lnc-DC controls human dendritic cell differentiation. Science 344: 310-313, 2014.

46. Ranzani V, Rossetti G, Panzeri I, Arrigoni A, Bonnal RJ, Curti S, Gruarin P, Provasi E, Sugliano E, Marconi M, et al: The long intergenic noncoding RNA landscape of human lymphocytes highlights the regulation of T cell differentiation by linc-MAF-4. Nat Immunol 16: 318-325, 2015.

This work is licensed under a Creative Commons Attribution-NonCommercial-NoDerivatives 4.0 International (CC BY-NC-ND 4.0) License. 